\title{
Usual Walking Speed Predicts Decline of Functional Capacity among Community- Dwelling Older Japanese Women: a 4-year Longitudinal Study
}

\author{
Hyuma Makizako, PhD, Msc, RPT ${ }^{1)}$, TAKeto Furuna, RPT ${ }^{2)}$, Hiroto Yoshida, $\mathrm{PhD}^{3)}$, \\ HIROYUKI SHIMADA, PhD, Msc, RPT ${ }^{1}$, KATSUNORI SATOH ${ }^{4}$, \\ TOMOMI AKANUMA, RPHN, RN ${ }^{4)}$, HIKARU IHIRA, Msc, RPT ${ }^{2)}$, TAKAO SUZUKI, PhD, MD ${ }^{5)}$ \\ ${ }^{1)}$ Section for Health Promotion, Department of Health and Medical Care, Center for \\ Development of Advanced Medicine for Dementia, National Center for Geriatrics and \\ Gerontology: 35 Gengo, Morioka-machi, Obu, Aichi 474-8511, Japan. \\ TEL: +81 562-46-2311, E-mail: makizako@ncgg.go.jp \\ ${ }^{2)}$ School of Health Sciences, Sapporo Medical University \\ ${ }^{3)}$ Tokyo Metropolitan Institute of Gerontology \\ 4) Bibai city office \\ ${ }^{5)}$ Research Institute, National Center for Geriatrics and Gerontology
}

\begin{abstract}
Purpose] In this study, we examined the physical predictors of the decline of functional capacity among community-dwelling older Japanese individuals. [Methods] A total of 265 Japanese individuals aged 68-96 years participated in a baseline survey and a follow-up 4 years later. In this survey, participants performed tests for grip strength, one-legged standing, and 5-m walking. At baseline and the follow-up survey, we measured functional capacity using the Tokyo Metropolitan Institute of Gerontology Index of Competence (TMIG-IC), which consists of the instrumental self-maintenance scale, the intellectual activity scale, and the social role scale. [Results] There was only a weak association between physical performance and the decline of functional status in men. The 5-m walking time was most sensitive at predicting the future decline of the instrumental self-maintenance scale in women. Even after controlling for age, body mass index, grip strength, and one-legged standing, a slower 5-m walking time (greater than $4.6 \mathrm{sec}$ ) indicated an increased risk of future decline in instrumental self-maintenance in women (odds ratio $=5.31$, $\mathrm{p}<0.01$ ). [Conclusions] The usual walking speed is the best performance measure for predicting the decline of instrumental self-maintenance among Japanese older women.
\end{abstract}

Key words: Aged, 5-m walking, Instrumental activities of daily living

(This article was submitted Apr. 2, 2010, and was accepted May 7, 2010)

\section{INTRODUCTION}

Good functional capacity has become an important key phrase for successful aging among the growing population of older people. Decline of functional capacity has been reported to correlate with a high mortality risk ${ }^{1)}$ and to predict placement in a nursing home ${ }^{2)}$. It is therefore important to determine predictors of decline and to maintain a high level of functional capacity among community-dwelling older individuals.

Previous studies have shown that performancebased measures of physical function predicted future incidence of disability, dependence in 
activities of daily living, and death in older individuals living independently in the community $^{3)}$. For instance, performance measures of lower-extremity function such as walking speed, standing balance and knee-extension strength, are important predictors of subsequent disability in the older population ${ }^{4-8)}$. Reduced hand-grip strength is another predictor of disability in older people ${ }^{9}$. However, none of these longitudinal studies have demonstrated cutoff points for performance-based measures of physical function to predict decline in functional capacity. Although most previous studies have found relationships between lower physical function and future decline in instrumental activities of daily living (IADL), other dimensions of functional capacity, including intellectual activity and social roles, have not been clearly identified.

The goal of the present study was to find physical predictors of decline in functional capacity and determine optimal cutoff points for physical performance measurements predicting decline in each element of functional capacity. In this study, we examined the best physical predictor of decline in functional capacity using the Tokyo Metropolitan Institute of Gerontology Index of Competence $(\mathrm{TMIG}-\mathrm{IC})^{10)}$, which contains three multidimensional subscales to evaluate older community-dwelling Japanese individuals. In addition, we determined cutoff points for physical performance measurements that were related to decline in functional capacity.

\section{SUBJECTS AND METHODS}

\section{Subjects}

A baseline survey including measurements of physical performance was carried out in the autumn of 2004 and 2005. First, all citizens aged 65 years and over living in Bibai-city, excluding those who were physically disabled or required long-term care, were asked to answer the self-reported TMIG-IC which was distributed by mail. A total of 5,193 people responded (74.0\% response), of these, 2,290 persons who showed some decline in the TMIG-IC were invited to participate in the baseline survey. A follow-up survey was conducted 4 years after the baseline survey using a self-report questionnaire. A total of 370 people aged 65 years and over completed the physical performance tests at baseline and 278 participants completed the self- report questionnaire at follow-up. We excluded subjects who reported the lowest score (i.e., a score of 0 ) on any of the TMIG-IC subscales at baseline (see next section in methods). Finally, we analyzed the data of 265 participants who had completed the physical performance tests at baseline and the selfreport questionnaire at follow-up and who did not have the lowest score on any TMIG-IC subscale at baseline. All data were collected in Bibai, a rural community in the middle of Hokkaido Prefecture, Japan. This study was approved by the ethics committee of the Tokyo Metropolitan Institute of Gerontology. All participants provided their written informed consent.

\section{Methods}

Functional capacity was measured using the TMIG-IC, a self-report questionnaire. This index is a multi-dimensional 13-item index that was developed to grade the functional capacity of elderly people with intact activities of daily living according to their abilities in complex activities (Table 1). This multi-dimensional index of competence is comprised of three subscales: instrumental self-maintenance, intellectual activity, and social roles. The reliability and validity of these subscales were previously confirmed in a large sample ${ }^{10)}$. The cross-validity of the TMIG-IC has been tested, in terms of construct validity and predictive validity, with two representative samples of Japanese elderly community residents ${ }^{11)}$. The response for each item is either 'yes' (able to do) or 'no' (unable), and each item is scored 1 for 'yes'or 0 for 'no'. A total score is created by simply summing the item scores. Thus, the total score in this index ranges from 0 to 13 points, and the subscales range from 0 to 5 points for instrumental self-maintenance, 0 to 4 points for intellectual activity, and 0 to 4 points for social roles. A high score indicates high functional capacity. We defined a decline in each subscale as a decrement of 1 or more points during the 4-year follow-up.

Participants performed tests for grip strength, one-legged standing, and 5-m walking. The grip strength of the preferred hand was measured using a hand-held Smedley-type dynamometer. The best result of two trials was recorded. One-legged standing is a commonly used balance assessment of postural stability. The participants were asked to stand on their preferred leg as long as possible with their arms hanging down and with their eyes open. 
Table 1. Items of the Tokyo Metropolitan Institute of Gerontology Index of Competence

\begin{tabular}{lll}
\hline Subscales & \multicolumn{2}{l}{ Questionnaire } \\
\hline Instrumental & 1 & Can you use public transportation (bus or train) by yourself? \\
self-maintenance & 2 & Are you able to shop for daily necessities? \\
& 3 & Are you able to prepare meals by yourself? \\
& 4 & Are you able to pay bills? \\
& 5 & Can you handle your own banking? \\
\hline Intellectual activity & 6 & Are you able to fill out forms for your pension? \\
& 7 & Do you read newspapers? \\
& 8 & Do you read books or magazines? \\
& 9 & Are you interested in news stories or programs dealing with health? \\
\hline Social role & 10 & Do you visit the homes of friends? \\
& 11 & Are you sometimes called on for advice? \\
& 12 & Are you able to visit sick friends? \\
& 13 & Do you sometimes initiate conversations with young people?
\end{tabular}

Note: The response to each item is either 'yes' (= able to do) or 'no' (= unable).

The total score is the number of items answered with 'yes'.

One-legged standing balance was measured as the time ( 0 to 60 seconds) participants could stand on one leg. The best time obtained out of two trials was recorded. The usual walking speed of the subjects was measured over an 11-m straight and level path. Time measured in seconds to pass the 5$m$ mark on the path was used as the subject's score. A 3-m approach was allowed before the starting marker, and an additional 3-m of space was provided after the end marker of the $5-\mathrm{m}$ path to ensure a usual walking pace throughout the task. Participants were instructed to walk the $11-\mathrm{m}$ path at their usual walking pace. The time to complete the 5-m walking test was measured once. The physical performance tests were conducted by Bibai office staff who had nursing, public health nursing or allied health qualifications. All staff received training from a clinical nurse in the correct protocols for administering all of the physical performance tests.

The results are expressed as mean \pm S.D. Unpaired $t$-test statistics were used for sample description. We also compared the gender differences in age, grip strength, one-legged standing time, and 5-m walking time between a declining group and a non-declining group of subjects for each subscale of the TMIG-IC using the unpaired $\mathrm{t}$-test. Odds ratios (ORs) were calculated separately for each gender using logistic regression analysis to evaluate which physical performance measurements correlated best with the future decline in functional capacity. Receiver operated characteristic (ROC) curves were used to determine optimal cutoff points for measurements of physical performance that best discriminated between those who did and did not report a decline in each of the TMIG-IC subscale during the 4-year follow-up period. We defined the cutoff value that equally balanced high sensitivity and specificity and the outcomes were grouped into two categories below and above the cutoff point. Finally, a multiple logistic regression analysis was performed to identify the relationship between the decline in functional capacity and the cutoff points for physical performance measurements. All statistical analyses were performed using SPSS $17.0 \mathrm{~J}$ for Windows (SPSS Inc., Chicago, IL). P-values $<0.05$ were considered statistically significant.

\section{RESULTS}

Compared to women, men showed significantly higher physical function in the three measurements of physical performance (Table 2). The numbers of participants who showed a decline in the instrumental self-maintenance scale, intellectual activity and social roles over the 4-year follow-up were $21(17.6 \%), 34(28.6 \%)$, and $43(36.1 \%)$ men and $29(20.0 \%), 36(24.7 \%)$, and $55(37.7 \%)$ women, respectively. Male subjects who experienced a decline in instrumental selfmaintenance had a significantly shorter one-legged standing time and a significantly slower $5-\mathrm{m}$ walking time than those in the non-declining group. 
Table 2. Sample description

\begin{tabular}{lcc}
\hline & Men & Women \\
\hline Number & 119 & 146 \\
Age (years) & $78.1 \pm 5.5$ & $78.2 \pm 5.9$ \\
& $68-96$ & $68-91$ \\
Body mass index $\left(\mathrm{kg} / \mathrm{m}^{2}\right)$ & $23.7 \pm 3.1$ & $23.9 \pm 3.3$ \\
\hline Baseline & & \\
TMIG-IC total score (range 0-13) & $11.0 \pm 1.3$ & $10.7 \pm 1.6$ \\
$\quad$ Instrumental self-maintenance (0-5) & $4.6 \pm 0.7$ & $4.7 \pm 0.7$ \\
Intellectual activities (0-4) & $3.5 \pm 0.7$ & $3.1 \pm 0.9 * *$ \\
$\quad$ Social roles (0-4) & $2.8 \pm 0.9$ & $2.9 \pm 0.9$ \\
Grip strength (kg) & $37.6 \pm 7.6$ & $23.3 \pm 5.4^{* *}$ \\
One-legged standing time (sec) & $32.4 \pm 23.3$ & $23.0 \pm 21.4 * *$ \\
5-m walking time (sec) & $4.2 \pm 1.0$ & $4.6 \pm 1.1 * *$ \\
\hline Follow-up & & \\
TMIG-IC total score (range 0-13) & $10.2 \pm 3.0$ & $10.1 \pm 3.0$ \\
Instrumental self-maintenance (0-5) & $4.4 \pm 1.2$ & $4.4 \pm 1.2$ \\
Intellectual activities (0-4) & $3.2 \pm 1.1$ & $3.1 \pm 1.2$ \\
Social roles (0-4) & $2.6 \pm 1.3$ & $2.7 \pm 1.3$ \\
\hline
\end{tabular}

Note: values are mean \pm standard deviation, or minimum-maximum. T-test for means, ${ }^{* *}: \mathrm{p}<0.01$. TMIG-IC $=$ Tokyo Metropolitan Institute of Gerontology Index of Competence.

In addition, those declining in intellectual activity had significantly shorter one-legged standing times than the non-declining group. Among female subjects, there were significant differences for age and all measurements of physical performance between the declining group and the non-declining group (Table 3).

Table 4 shows the ORs determined by logistic regression analysis for baseline variables with respect to decline in each dimension of functional capacity during the 4-year follow-up period. There were no significant associations between physical performance and decline of functional status in men. The 5-m walking time was most sensitive at predicting the future decline of instrumental selfmaintenance in women. However, in the logistic regression analysis, none of the physical performance tests were found to be significantly associated with subsequent declines in intellectual activities or social roles in women.

We used ROC analysis to determine optimal cutoff points for the 5-m walking time between women who did and did not report a decline in instrumental self-maintenance during the 4-year follow-up period (Fig. 1). The area under the curve (AUC) of the 5-m walking time was 0.81 [95\% confidence interval $(\mathrm{CI}): 0.72$ to $0.91, \mathrm{p}<0.01]$. As shown on Fig. 1, the two points closest to the upper left corner of the ROC curve are the times $\geq 5.4 \mathrm{sec}$, with $65.5 \%$ sensitivity and an $88.9 \%$ specificity, and $\geq 4.6 \mathrm{sec}$, with $82.8 \%$ sensitivity and $70.1 \%$ specificity. Using this screening measurement for preventing decline in instrumental selfmaintenance, a cutoff point of $\geq 4.6 \mathrm{sec}$ was chosen because of its higher sensitivity ( $82.8 \%$ ). In Model 1 of the multiple logistic regression analysis, when adjusting for age and body mass index, a walking time slower than the cutoff point of $\geq 4.6 \mathrm{sec}$ was significantly correlated with decline in instrumental self-maintenance during the 4-year follow-up period (ORs $=7.18,95 \%$ CI: 2.33 to $22.16, \mathrm{p}<0.01$ ) (Table 5). Even after controlling for age, body mass index, grip strength, and one-legged standing, a slower 5-m walking time ( $\geq 4.6 \mathrm{sec})$ indicated an increased risk of decline in instrumental selfmaintenance in women $(\mathrm{ORs}=5.31,95 \% \mathrm{CI}: 1.63$ to $17.22, \mathrm{p}<0.01$ ).

\section{DISCUSSION}

We found that the 5-m walking time was the best performance measure for predicting the decline of instrumental self-maintenance among older Japanese women. In particular, older women who 
Table 3. Comparison of baseline physical performances between subjects with and without decline of higher-level functional capacity over the 4-year follow-up period

\begin{tabular}{|c|c|c|c|c|c|c|}
\hline & \multicolumn{6}{|c|}{$\operatorname{Men}(n=119)$} \\
\hline & \multicolumn{2}{|c|}{ Instrumental self-maintenance } & \multicolumn{2}{|c|}{ Intellectual activities } & \multicolumn{2}{|c|}{ Social roles } \\
\hline & $\begin{array}{l}\text { decline } \\
(\mathrm{n}=21)\end{array}$ & $\begin{array}{l}\text { non-decline } \\
(\mathrm{n}=98)\end{array}$ & $\begin{array}{l}\text { decline } \\
(\mathrm{n}=34)\end{array}$ & $\begin{array}{l}\text { non-decline } \\
(\mathrm{n}=85)\end{array}$ & $\begin{array}{l}\text { decline } \\
(\mathrm{n}=43)\end{array}$ & $\begin{array}{l}\text { non-decline } \\
(\mathrm{n}=76)\end{array}$ \\
\hline Age (years) & $79.1 \pm 4.5$ & $77.8 \pm 5.7$ & $78.9 \pm 5.9$ & $77.7 \pm 5.4$ & $79.0 \pm 5.3$ & $77.5 \pm 5.6$ \\
\hline Grip strength $(\mathrm{kg})$ & $35.4 \pm 8.8$ & $38.0 \pm 7.3$ & $36.2 \pm 8.9$ & $38.1 \pm 6.9$ & $36.8 \pm 7.2$ & $38.0 \pm 7.8$ \\
\hline One-legged standing time (sec) & $21.4 \pm 23.5$ & $34.7 \pm 22.7^{*}$ & $26.4 \pm 23.7$ & $34.8 \pm 22.9^{*}$ & $29.3 \pm 24.3$ & $34.1 \pm 22.7$ \\
\hline \multirow[t]{4}{*}{ 5-m walking time $(\mathrm{sec})$} & $4.6 \pm 1.3$ & $4.1 \pm 0.8^{*}$ & $4.4 \pm 1.1$ & $4.1 \pm 0.9$ & $4.3 \pm 1.0$ & $4.1 \pm 0.9$ \\
\hline & \multicolumn{6}{|c|}{ Women $(n=146)$} \\
\hline & \multicolumn{2}{|c|}{ Instrumental self-maintenance } & \multicolumn{2}{|c|}{ Intellectual activities } & \multicolumn{2}{|c|}{ Social roles } \\
\hline & $\begin{array}{l}\text { decline } \\
(\mathrm{n}=29)\end{array}$ & $\begin{array}{l}\text { non-decline } \\
(\mathrm{n}=117)\end{array}$ & $\begin{array}{l}\text { decline } \\
(\mathrm{n}=36)\end{array}$ & $\begin{array}{l}\text { non-decline } \\
(\mathrm{n}=110)\end{array}$ & $\begin{array}{l}\text { decline } \\
(\mathrm{n}=55)\end{array}$ & $\begin{array}{l}\text { non-decline } \\
(\mathrm{n}=91)\end{array}$ \\
\hline Age (years) & $82.3 \pm 4.5$ & $77.2 \pm 5.8 * *$ & $81.5 \pm 5.6$ & $77.1 \pm 5.6^{* *}$ & $80.3 \pm 5.5$ & $76.9 \pm 5.8 * *$ \\
\hline Grip strength (kg) & $19.4 \pm 5.5$ & $24.2 \pm 5.0 * *$ & $20.7 \pm 4.9$ & $24.1 \pm 5.3^{* *}$ & $21.7 \pm 5.5$ & $24.2 \pm 5.1 * *$ \\
\hline One-legged standing time (sec) & $11.5 \pm 12.4$ & $25.8 \pm 22.3^{* *}$ & $14.4 \pm 16.3$ & $25.8 \pm 22.2^{* *}$ & $16.9 \pm 18.52$ & $26.7 \pm 22.3 * *$ \\
\hline 5-m walking time (sec) & $5.6 \pm 1.2$ & $4.3 \pm 0.9 * *$ & $5.2 \pm 1.3$ & $4.4 \pm 0.9 * *$ & $4.9 \pm 1.3$ & $4.4 \pm 0.9 * *$ \\
\hline
\end{tabular}

Note: t-test for means, $*: \mathrm{p}<0.05, * *: \mathrm{p}<0.01$.

Table 4. Odds ratios of decline in each dimension of functional capacity during the 4-year follow-up period for each of the baseline variables

\begin{tabular}{|c|c|c|c|c|c|c|}
\hline \multirow[b]{3}{*}{ Variables } & \multicolumn{6}{|c|}{ Men } \\
\hline & \multicolumn{2}{|c|}{ Instrumental self-maintenance } & \multicolumn{2}{|c|}{ Intellectual activities } & \multicolumn{2}{|c|}{ Social roles } \\
\hline & OR & $95 \% \mathrm{CI}$ & OR & $95 \% \mathrm{CI}$ & OR & $95 \% \mathrm{CI}$ \\
\hline Age & 0.97 & $0.87-1.07$ & 1.00 & $0.91-1.09$ & 1.04 & $0.96-1.13$ \\
\hline Body mass index & 0.91 & $0.75-1.09$ & 1.03 & $0.90-1.19$ & 1.00 & $0.87-1.14$ \\
\hline Grip strength & 0.99 & $0.92-1.08$ & 0.98 & $0.91-1.05$ & 1.00 & $0.94-1.07$ \\
\hline One-legged standing time & 0.98 & $0.95-1.00$ & 0.99 & $0.97-1.01$ & 1.00 & $0.98-1.02$ \\
\hline \multirow[t]{3}{*}{ 5-m walking time } & 1.58 & $0.94-2.63$ & 1.15 & $0.74-1.79$ & 1.08 & $0.70-1.66$ \\
\hline & \multicolumn{6}{|c|}{ Women } \\
\hline & \multicolumn{2}{|c|}{ Instrumental self-maintenance } & \multicolumn{2}{|c|}{ Intellectual activities } & \multicolumn{2}{|c|}{ Social roles } \\
\hline Variables & OR & $95 \% \mathrm{CI}$ & OR & $95 \% \mathrm{CI}$ & OR & $95 \% \mathrm{CI}$ \\
\hline Age & 1.02 & $0.91-1.14$ & 1.06 & $0.96-1.17$ & 1.05 & $0.96-1.14$ \\
\hline Body mass index & 0.95 & $0.83-1.10$ & 1.04 & $0.92-1.18$ & 0.96 & $0.86-1.08$ \\
\hline Grip strength & 0.92 & $0.82-1.03$ & 0.95 & $0.86-1.05$ & 0.97 & $0.89-1.06$ \\
\hline One-legged standing time & 0.98 & $0.95-1.02$ & 0.99 & $0.97-1.02$ & 0.99 & $0.97-1.01$ \\
\hline 5-m walking time & $2.20 * *$ & $1.30-3.71$ & 1.44 & $0.93-2.25$ & 1.18 & $0.79-1.77$ \\
\hline
\end{tabular}

Note: $\mathrm{OR}=$ odds ratio, $\mathrm{CI}=$ confidence intervals, $* *: \mathrm{p}<0.01$.

Table 5. Odds ratios of decline in IADL during the 4-year follow-up among women subjects $(\mathrm{n}=146)$ for above and below the cutoff point of 5-m walking time

\begin{tabular}{|c|c|c|c|c|c|}
\hline \multirow[b]{2}{*}{ 5-m walking time } & \multirow[b]{2}{*}{ Number $(\%)$} & \multicolumn{2}{|c|}{ Model 1 ${ }^{\mathrm{a}}$} & \multicolumn{2}{|c|}{ Model $2^{b}$} \\
\hline & & OR & $95 \% \mathrm{CI}$ & OR & $95 \% \mathrm{CI}$ \\
\hline$\leq 4.5 \mathrm{sec}$ & $87(59.6)$ & 1.00 & Reference & 1.00 & Reference \\
\hline$\geq 4.6 \mathrm{sec}$ & $59(40.4)$ & $7.18 * *$ & $2.33-22.16$ & $5.31 * *$ & $1.63-17.22$ \\
\hline
\end{tabular}

Note: $\mathrm{OR}=$ odds ratio, $\mathrm{CI}=$ confidence intervals, $* *: \mathrm{p}<0.01$. ${ }^{\text {a }}$ Model $1=$ adjusted for age and body mass index. ${ }^{\mathrm{b}}$ Model 2 = adjusted for age, body mass index, grip strength, and one-legged standing time. 


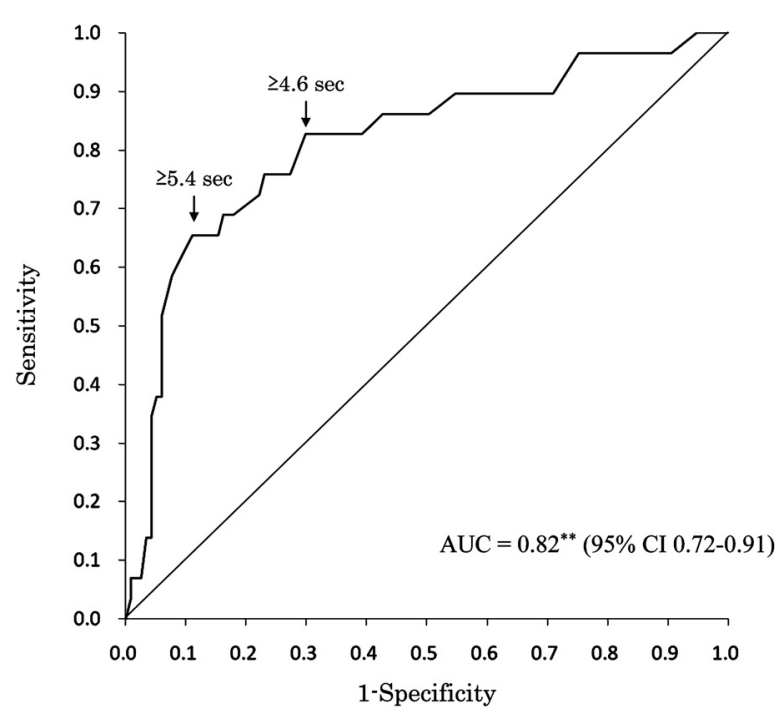

Fig. 1. Receiver operating characteristic (ROC) curve of 5$\mathrm{m}$ walking time used to detect older women at risk of subsequent decline in instrumental selfmaintenance during the 4-year follow-up period. Note: $\mathrm{AUC}=$ the area under the ROC curve, $\mathrm{CI}=$ confidence intervals, $* *$ : $\mathrm{p}<0.01$.

had a poor walking performance ( $\geq 4.6 \mathrm{sec}$ over 5 $\mathrm{m}$ ) had a 5.31-fold higher risk of showing a decline in instrumental self-maintenance during the 4-year follow-up period. This remained significant even after controlling for age, body mass index, grip strength, and one-legged standing time. However, we found that physical performance was not statistically related to the future decline in intellectual activities or social roles in older women. In addition, there was only a weak association between physical performance and the decline of functional status in older men.

The measurement of walking time has been suggested to be a useful test of physical function in clinical settings ${ }^{12}$. It has been reported that the walking speed of the older population is associated with performance in standardized tests of physical function ${ }^{13)}$. Our results indicate that the walking speed is a good performance measure for predicting the decline of IADL among older Japanese women, similar to previous studies ${ }^{4,5)}$. However, for male subjects there was only a weak association between the 5-m walking time and decline in instrumental self-maintenance, and no physical performance test was significantly associated with a subsequent decline in intellectual activities or social roles. Poor intellectual and social activities were found to be factors predicting a decline in IADL ${ }^{14,15)}$. Thus, intellectual and social activities may have declined ahead of the onset of instrumental self-maintenance decline among our subjects, consistent with the findings of a previous study ${ }^{16)}$. In fact, we found higher percentages in the decline of intellectual activities and social roles in both men and women compared to the declines in instrumental selfmaintenance (Table 3). Other factors such as depressive symptoms ${ }^{17)}$, cognitive status ${ }^{18)}$ and self-rated health ${ }^{19)}$ may be significantly related to the risk of decline in intellectual activities and social roles rather than the physical performance of subjects. Especially, these factors may influence the changes in IADL among older men. Therefore, there might be only a weak association between physical performance tests and decline of instrumental self-maintenance in male subjects.

The most significant result from our study was the identification of a potential cutoff point for the 5 -m walking time. We found that older women who took longer than $4.6 \mathrm{sec}$ to walk $5 \mathrm{~m}$ were at a 5.31fold higher risk of future decline in instrumental self-maintenance at the 4-year follow-up period. The $4.6 \mathrm{sec}$ cutoff had a sensitivity of $82.8 \%$ and a specificity of $70.1 \%$. A slow usual walking speed in older individuals has a high predictive value for health-related events ${ }^{20)}$. One longitudinal study reported that the age-related decline in usual walking speed increased with advancing age among Japanese women ${ }^{21)}$. Physical performance measures, especially usual walking performance in older women, are important and essential tests for predicting future changes in IADL among the Japanese older population.

This study had several limitations. There were limited clinical assessments available to explain the relationship between the change in physical performance and functional capacity. For instance, some epidemiological investigations have shown that cognitive status and chronic medical conditions are good predictors of changes in IADL among community-dwelling older people ${ }^{22-24)}$. Also, we did not investigate social factors which may be related to decline in functional capacity (e.g., family relations, income, and social supports). Furthermore, these findings are based on a population of individuals who had already reported some functional limitations at baseline, even though we excluded those with the lowest score on any TMIG-IC subscale at the baseline. Also, we did not 
include enough individuals to make relevant comparisons with previous longitudinal observational findings.

In conclusion, this longitudinal study confirmed that the usual walking speed is the best performance measure for predicting the decline of instrumental self-maintenance among older Japanese women. In addition, even after controlling for age, body mass index, grip strength, and one-legged standing time, a slower $5-\mathrm{m}$ walking time $(\geq 4.6 \mathrm{sec})$ showed a 5.31 -fold increased risk for the decline of instrumental self-maintenance in older Japanese women. The test of usual walking performance is a simple measure and should be formally assessed to identify those at risk of subsequent decline in IADL among community-dwelling older individuals.

\section{ACKNOWLEDGEMENTS}

We would like to thank the staff in Bibai, Hokkaido, Japan for their help with the survey and data collection for this study. This study was supported by grants from the Ministry of Health, Labour, and Welfare of Japan.

\section{REFERENCES}

1) Ramos LR, Simoes EJ, Albert MS: Dependence in activities of daily living and cognitive impairment strongly predicted mortality in older urban residents in Brazil: a 2-year follow-up. J Am Geriatr Soc, 2001, 49: 1168-1175.

2) Wolinsky FD, Callahan CM, Fitzgerald JF, et al.: Changes in functional status and the risks of subsequent nursing home placement and death. J Gerontol, 1993, 48: S94-101.

3) Guralnik JM, Simonsick EM, Ferrucci L, et al.: A short physical performance battery assessing lower extremity function: association with self-reported disability and prediction of mortality and nursing home admission. J Gerontol, 1994, 49: M85-M94.

4) Suzuki T, Yoshida H, Kim H, et al.: Walking speed as a good predictor for maintenance of I-ADL among the rural community elderly in Japan: A 5-year follow-up study from TMIG-LISA. Geriatr Gerontol Int, 2003, 3: S6-S14.

5) Shinkai S, Watanabe S, Kumagai S, et al.: Walking speed as a good predictor for the onset of functional dependence in a Japanese rural community population. Age Aging, 2000, 29: 441-446.

6) Guralnik JM, Ferrucci L, Simonsick EM, et al.: Lowerextremity function in persons over the age of 70 years as a predictor of subsequent disability. N Engl J Med, 1995, 332: 556-561.
7) Rantanen T, Guralnik JM, Ferrucci L, et al.: Coimpairments as predictors of severe walking disability in older women. J Am Geriatr Soc, 2001, 49: 21-27.

8) Onder G, Penninx BW, Ferrucci L, et al.: Measures of physical performance and risk for progressive and catastrophic disability: results from the Women's Health and Aging Study. J Gerontol A Biol Sci Med Sci, 2005, 60: 74-79.

9) Giampaoli S, Ferrucci L, Cecchi F, et al.: Hand-grip strength predicts incident disability in non-disabled older men. Age Ageing, 1999, 28: 283-288.

10) Koyano W, Shibata H, Nakazato $K$, et al.: Measurement of competence: reliability and validity of the TMIG Index of Competence. Arch Gerontol Geriatr, 1991, 13: 103-116.

11) Koyano W, Shibata H: Cross-validation of the TMIG Index of Competence: invariability of factor structure and predictive validity. Japanese Journal of Gerontology, 1992, 14: 34-42 (in Japanese).

12) Shimada H, Suzukawa M, Tiedemann A, et al.: Which neuromuscular or cognitive test is the optimal screening tool to predict falls in frail communitydwelling older people? Gerontology, 2009, 55: 532538.

13) Nagasaki $H$, Itoh $H$, Furuna $T$ : The structure underlying physical performance measures for older adults in the community. Aging Clin Exp Res, 1995, 7: 451-458.

14) Shinkai S, Kumagai S, Fujiwara Y, et al.: Predictors for the onset of functional decline among initially nondisabled older people living in a community during a 6-year follow-up. Geriatr Gerontol Int, 2003, 3: 31-39.

15) Ishizaki T, Watanabe S, Suzuki T, et al.: Predictors for functional decline among nondisabled older Japanese living in a community during a 3-year follow-up. J Am Geriatr Soc, 2000, 48: 1424-1429.

16) Fujiwara $Y$, Shinkai S, Kumagai S, et al.: Longitudinal changes in higher-level functional capacity of an older population living in a Japanese urban community. Arch Gerontol Geriatr, 2003, 36: 141-153.

17) Kondo N, Kazama M, Suzuki K, et al.: Impact of mental health on daily living activities of Japanese elderly. Prev Med, 2008, 46: 457-462.

18) Bassuk SS, Glass TA, Berkman LF: Social disengagement and incident cognitive decline in community-dwelling elderly persons. Ann Intern Med, 1999, 131: 165-173.

19) Lee HY, Jang SN, Lee S, et al.: The relationship between social participation and self-rated health by sex and age: a cross-sectional survey. Int J Nurs Stud, 2008, 45: 1042-1054.

20) Cesari M, Kritchevsky SB, Penninx BW, et al.: Prognostic value of usual gait speed in wellfunctioning older people-results from the Health, Aging and Body Composition Study. J Am Geriatr Soc, 2005, 53: 1675-1680.

21) Furuna $T$, Nagasaki $H$, Nishizawa $S$, et al.: 
Longitudinal change in the physical performance of older adults in the community. J Jpn Phys Ther Assoc, 1998, 1: 1-5.

22) Fujiwara $Y$, Shinkai S, Kumagai S, et al.: Impact of history or onset of chronic medical conditions on higher-level functional capacity among older community-dwelling Japanese adults. Geriatr Gerontol Int, 2003, 3: 69-77.

23) Fujiwara Y, Yoshida H, Amano H, et al.: Predictors of improvement or decline in instrumental activities of daily living among community-dwelling older Japanese. Gerontology, 2008, 54: 373-380.

24) Dodge $\mathrm{HH}$, Kadowaki $\mathrm{T}$, Hayakawa $\mathrm{T}$, et al.: Cognitive impairment as a strong predictor of incident disability in specific ADL-IADL tasks among community-dwelling elders: the Azuchi Study. Gerontologist, 2005, 45: 222-230. 